\title{
THEORETICAL STUDY OF FAST ELECTRON-IMPACT IONIZATION OF POLARIZED ATOMS *
}

\author{
K. Glemža ${ }^{\mathrm{a}}$ and A. Kupliauskiené ${ }^{\mathrm{b}}$ \\ ${ }^{a}$ Faculty of Physics, Vilnius University, Saulètekio 9, LT-10222 Vilnius, Lithuania \\ E-mail: kazimieras.glemza@vu.ff.lt \\ ${ }^{\mathrm{b}}$ Institute of Theoretical Physics and Astronomy of Vilnius University, A.Goštauto 12, LT-01108 Vilnius, Lithuania \\ E-mail: akupl@itpa.lt
}

Received 7 September 2005

\begin{abstract}
In the first Born approximation, the general expression for ionization cross-section of polarized atoms by fast non-polarized electrons is derived by using the methods of the theory of an atom adapted for polarization and the similarity between the Born and photoionization operators. Special cases of the general expression for description of the angular distribution of the slower emitted electron and for the alignment of ionized atoms are obtained in the case of polarized and non-polarized atoms, as well as the magnetic dichroism of the total ionization cross-section of polarized atoms.
\end{abstract}

Keywords: ionization of atoms by electron impact, polarization, angular distribution

PACS: $34.80 . \mathrm{Dp}, 29.25 . \mathrm{Pj}$, 29.25.Lg

\section{Introduction}

The interaction of atoms with photons, electrons, and other charged particles is very important process in laboratory and astrophysical plasmas resulting in the distortion of Maxwellian distribution of electron velocities [1]. The non-equilibrium population of magnetic sublevels or the ordering of angular momenta of atomic particles that is called a self-alignment arises in these processes. The polarization of the emitted electromagnetic radiation could be considered as an indication of the presence of ordered beams of electrons in plasma. Recently, the methods of the theory of an atom were applied for the derivation of the expressions describing the interaction of polarized photons and electrons with polarized atoms and ions [2-6]. The probability or cross-section of the interaction was expressed as the multiple expansion over the multipoles (irreducible tensors) of the state of all particles taking part in the process both in initial and final states. The applied approach was an alternative to the density matrix method [7] where the density matrix was expressed via multipoles or statistical tensors.

The ionization of polarized atoms by polarized electrons was investigated by Kupliauskienè and Glemža

\footnotetext{
* The report presented at the 36th Lithuanian National Physics Conference, 16-18 June 2005, Vilnius, Lithuania.
}

[5] with the help of the method of the theory of an atom [2] in distorted wave approximation. In the case of ionization of atoms by fast non-polarized electrons a simpler approach like plane wave Born approximation (PWBA) can be applied [8-10]. This approximation allows us to describe the angular distribution and spin polarization of the slower electron emitted from a polarized or non-polarized atom [9], as well as the alignment of the ionized atoms $[8,9]$. The description of orientational dichroism in the electron-impact ionization of laser-oriented atoms [10] is also possible.

The main task of the present work is the derivation of a general expression for the non-polarized electronimpact ionization of polarized atoms in non-relativistic approximation, the special cases of which were suitable for the interpretation of the experimental results [10]. The next section of the present work is devoted to obtaining the general expression. Its special cases are presented in Section 3. The inequality

$$
\begin{aligned}
& \text { fine structure splitting } \\
& \gg \text { line width } \\
& \gg \text { hyperfine structure splitting }
\end{aligned}
$$

is also assumed. The modifications enabling one to take into account the hyperfine structure splitting can be easily made $[2,4]$. 


\section{General expression}

The process of ionization of an atom $A$ in the state $\alpha_{1} J_{1} M_{1}$ by an electron $e^{-}$moving with the momentum $\mathbf{p}_{1}$ can be written as follows:

$$
\begin{aligned}
& A\left(\alpha_{1} J_{1} M_{1}\right)+e^{-}\left(\mathbf{p}_{1} m_{1}\right) \\
& \rightarrow A^{+}\left(\alpha_{2} J_{2} M_{2}\right)+e^{-}(\mathbf{p} m)+e^{-}\left(\mathbf{p}_{2} m_{2}\right),
\end{aligned}
$$

where $A^{+}$denotes the ion in the state $\alpha_{2} J_{2} M_{2}$. In (1), $\alpha_{1}\left(\alpha_{2}\right)$ indicates the configuration and other quantum numbers, $J_{1}\left(J_{2}\right)$ and $M_{1}\left(M_{2}\right)$ are total angular momentum and its projection of an atom (ion), respectively, $\mathbf{p}_{2}$ and $\mathbf{p}$ stand for the momenta of the scattered and emitted electrons, respectively, and $m_{1}, m_{2}$, and $m$ indicate the projections of spins of the projectile, scattered, and emitted electrons, respectively. In general, the projections of angular momenta of all the particles taking part in the process (1) can be defined onto different directions. The fine structure splitting is assumed to be much larger than the hyperfine one. Then the states of atom and ion can be specified by the total angular momentum of all electronic shells.

In the first order of perturbation theory, the probability of ionization of an atom (1) can be written as follows:

$$
\begin{aligned}
& \frac{\mathrm{d} W\left(\alpha_{1} J_{1} M_{1} \mathbf{p}_{1} m_{1} \rightarrow \alpha_{2} J_{2} M_{2} \mathbf{p} m \mathbf{p}_{2} m_{2}\right)}{\mathrm{d} \varepsilon \mathrm{d} \Omega \mathrm{d} \Omega_{2}} \\
& =C\left\langle\alpha_{2} J_{2} M_{2} \mathbf{p} m \mathbf{p}_{2} m_{2}|H| \alpha_{1} J_{1} M_{1} \mathbf{p}_{1} m_{1}\right\rangle \\
& \quad \times\left\langle\alpha_{2} J_{2} M_{2} \mathbf{p} m \mathbf{p}_{2} m_{2}|H| \alpha_{1} J_{1} M_{1} \mathbf{p}_{1} m_{1}\right\rangle^{*} \\
& \quad \times \delta\left(E_{1}-E_{2}\right) .
\end{aligned}
$$

Here and below the atomic system of units is used. In (2), $H$ is the operator of the electrostatic interaction between the projectile and atomic electrons, $E_{1}$ and $E_{2}$ are the energies of the systems atom + electron in the initial state and ion + two electrons in the final state, respectively, $C$ is the constant depending on the normalization of the wave function of free electron.

When the projectile and scattered electrons are described by plane waves normalized to unity, the matrix element in (2) can be written in the form

$$
\begin{aligned}
& \left\langle\left(\alpha_{2} J_{2} M_{2} \mathbf{p} m\right) \mathbf{p}_{2}|H| \alpha_{1} J_{1} M_{1} \mathbf{p}_{1}\right\rangle \\
& =\left\langle\left(\alpha_{2} J_{2} M_{2} \mathbf{p} m\right) \mathrm{e}^{-\mathrm{i} \mathbf{k}_{2} \mathbf{r}_{e}}\right| \\
& \quad \times \sum_{j} \frac{1}{\left|\mathbf{r}_{j}-\mathbf{r}_{e}\right|}\left|\alpha_{1} J_{1} M_{1} \mathrm{e}^{\mathrm{i} \mathbf{k}_{1} \mathbf{r}_{e}}\right\rangle
\end{aligned}
$$

$$
\begin{aligned}
& =\left\langle\alpha_{2} J_{2} M_{2} \mathbf{p} m\left|\sum_{j} \mathrm{e}^{\mathrm{iq \mathbf {r }}} \frac{1}{\left|\mathbf{r}_{j}-\mathbf{r}_{e}\right|}\right| \alpha_{1} J_{1} M_{1}\right\rangle \\
& =\frac{4 \pi}{q^{2}}\left\langle\alpha_{2} J_{2} M_{2} \mathbf{p} m\left|\sum_{j} \mathrm{e}^{\mathrm{iq} \mathbf{r}_{j}}\right| \alpha_{1} J_{1} M_{1}\right\rangle .
\end{aligned}
$$

Here $\mathbf{k}=\mathbf{p} / \hbar$, the transferred momentum is equal to $\mathbf{q}=\mathbf{k}_{1}-\mathbf{k}_{2}$, and the summation over the projections of the spins of free electrons is carried out. For the exponent in (3), the expansion

$$
\begin{aligned}
\mathrm{e}^{\mathrm{i} \mathbf{q} \mathbf{r}} & =4 \pi \sum_{t=0}^{\infty} \mathrm{i}^{t} j_{t}(q r) \sum_{p=-t}^{t} Y_{t p}^{*}(\hat{q}) Y_{t p}(\hat{r}) \\
& =\sum_{t=0}^{\infty} \sqrt{4 \pi(2 t+1)} \mathrm{i}^{t} j_{t}(q r) \\
& \times \sum_{p=-t}^{t} Y_{t p}^{*}(\hat{q}) C_{p}^{(t)}(\hat{r})
\end{aligned}
$$

is applied. The partial wave expansion can be used for the emitted electron:

$$
\begin{aligned}
|\mathbf{p} m\rangle & =4 \pi \sum_{\lambda \mu} R_{\lambda}(r) Y_{\lambda \mu}(\hat{r}) Y_{\lambda \mu}^{*}(\hat{p}) \xi_{m}(\sigma) \\
& =\sum_{\lambda \mu} \sqrt{4 \pi(2 \lambda+1)} R_{\lambda}(r) C_{\mu}^{(\lambda)}(\hat{r}) Y_{\lambda \mu}^{*}(\hat{p}) \xi_{m}(\sigma) .
\end{aligned}
$$

Here $\xi_{m}(\sigma)$ is a spin function of an electron, and

$$
R_{\lambda}(r)=\mathrm{i}^{\lambda} \exp \left[\mathrm{i}\left(\sigma_{\lambda}(p)+\delta_{\lambda}\right)\right] r^{-1} P(\varepsilon \lambda \mid r),
$$

where $P(\varepsilon \lambda \mid r)$ is a Hartree radial orbital of the electron in the continuum. The substitution of (4) and (5) into (3) leads to the following expression:

$$
\begin{aligned}
& \left\langle\alpha_{2} J_{2} M_{2} \mathbf{p} m \mathbf{q}|H| \alpha_{1} J_{1} M_{1}\right\rangle \\
& =\frac{(4 \pi)^{2}}{q^{2}} \sum_{t=0}^{\infty} \sum_{\lambda=0}^{\infty}[(2 \lambda+1)(2 t+1)]^{1 / 2}\left\langle\alpha_{2} J_{2} M_{2} \varepsilon \lambda\right| \\
& \quad \times \sum_{j^{\prime}} \mathrm{i}^{t} j_{t}(q r) C_{0}^{(t)}\left(\hat{r}_{j^{\prime}}\right)\left|\alpha_{1} J_{1} M_{1}\right\rangle Y_{t 0}(\hat{q}) Y_{\lambda 0}^{*}(\hat{p}) .(7)
\end{aligned}
$$

The second subscripts in $Y_{t 0}(\hat{q})$ and $Y_{\lambda 0}^{*}(\hat{p})$ are equal to zero because they are defined with respect to the quantization axes $z$ coinciding with the directions of transferred momentum and movement of emitted electron, respectively. The projections of the angular momenta $J_{1}$ and $J_{2}$ are also chosen arbitrarily. The calculation of the matrix element should be performed by using the 
projections defined with respect to the same quantization axis $z$ by rotation procedure. Mathematically it can be accomplished with the help of relation

$$
|j \tilde{m}\rangle=\sum_{m} D_{m \tilde{m}}^{j}(\alpha, \beta, \gamma)|j m\rangle,
$$

where $D_{m \tilde{m}}^{j}(\alpha, \beta, \gamma)$ is the Wigner rotation matrix. Then the matrix element (7) acquires the following expression:

$$
\begin{aligned}
& \left\langle\left(\alpha_{2} J_{2} M_{2} \mathbf{p} m\right) \mathbf{q}|H| \alpha_{1} J_{1} M_{1}\right\rangle \\
& =\frac{(4 \pi)^{2}}{q^{2}} \sum_{t=0}^{\infty} \sum_{\lambda}[(2 \lambda+1)(2 t+1)]^{1 / 2} \\
& \quad \times \sum_{\tilde{M}_{1}, \tilde{M}_{2}, m_{t}, \mu, \tilde{m}}\left\langle\alpha_{2} J_{2} \tilde{M}_{2} \varepsilon \lambda \mu\right| \\
& \quad \times \mathrm{i}^{t} j_{t}(q r) C_{m_{t}}^{(t)}(\hat{r})\left|\alpha_{1} J_{1} \tilde{M}_{1}\right\rangle D_{\tilde{M}_{1} M_{1}}^{J_{1}}\left(\hat{J}_{1}\right) \\
& \quad \times D_{\tilde{M}_{2} M_{2}}^{* J_{2}}\left(\hat{J}_{2}\right) D_{m_{t} 0}^{t}(\hat{q}) D_{\mu 0}^{* \lambda}(\hat{p}) D_{\tilde{m} m}^{* s}(\hat{s}) .
\end{aligned}
$$

The inspection of (9) allows us to notice that the angular part of this matrix element coincides with that of photoionization [4]. For this reason the same angular momentum diagrams can be used for the integration over orbital and spin angular momenta in (9). The final expression for the triply differential cross-section is as follows:

$$
\begin{aligned}
& \frac{\mathrm{d}^{3} \sigma\left(\alpha_{1} J_{1} M_{1} \mathbf{q} \rightarrow \alpha_{2} J_{2} M_{2} \mathbf{p} m\right)}{\mathrm{d} \Omega \mathrm{d} \Omega_{q} \mathrm{~d} \varepsilon}=\frac{C k_{2} k}{q^{4} k_{1}} \\
& \times \sum_{\substack{K_{1}, K_{2}, K_{q}, K_{\lambda}, K_{s}, K_{j}, K}} \mathcal{B}^{\text {B.ion. }}\left(K_{1}, K_{2}, K_{q}, K_{\lambda}, K_{s}, K_{j}, K\right) \\
& \times \sum_{\substack{N_{1}, N_{2}, N_{q}, N_{\lambda}, N_{s}, N_{j}, N}}\left[\begin{array}{l}
K_{1} K_{q} K \\
N_{1} N_{q} N
\end{array}\right]\left[\begin{array}{l}
K_{\lambda} K_{s} K_{j} \\
N_{\lambda} N_{s} N_{j}
\end{array}\right]\left[\begin{array}{l}
K_{2} K_{j} K \\
N_{2} N_{j} N
\end{array}\right] \\
& \times \sqrt{4 \pi} Y_{K_{\lambda} N_{\lambda}}(\hat{p}) \sqrt{4 \pi} Y_{K_{q} N_{q}}^{*}(\hat{q}) \\
& \times T_{N_{1}}^{* K_{1}}\left(\hat{J}_{1}\right) T_{N_{2}}^{K_{2}}\left(\hat{J}_{2}\right) T_{N_{s}}^{K_{s}}(\hat{s}),
\end{aligned}
$$

where

$$
\begin{aligned}
& \mathcal{B}^{\text {B.ion. }}\left(K_{1}, K_{2}, K_{q}, K_{\lambda}, K_{s}, K_{j}, K\right) \\
& =\sum_{\lambda, j, J, \lambda^{\prime}, j^{\prime}, J^{\prime}, t, t^{\prime}}(2 J+1)\left(2 J^{\prime}+1\right)(-1)^{\lambda^{\prime}+t^{\prime}}
\end{aligned}
$$

$$
\begin{aligned}
& \times\left\langle\alpha_{2} J_{2} \varepsilon \lambda(j) J\left\|Q^{(t)}\right\| \alpha_{1} J_{1}\right\rangle\left[\left(2 J_{1}+1\right)\left(2 J_{2}+1\right)\right]^{1 / 2} \\
& \times\left\langle\alpha_{2} J_{2} \varepsilon \lambda^{\prime}\left(j^{\prime}\right) J^{\prime}\left\|Q^{\left(t^{\prime}\right)}\right\| \alpha_{1} J_{1}\right\rangle^{*}[(2 s+1)]^{1 / 2} \\
& \times\left[\begin{array}{ccc}
t & t^{\prime} & K_{q} \\
0 & 0 & 0
\end{array}\right]\left[\begin{array}{ccc}
\lambda & \lambda^{\prime} & K_{\lambda} \\
0 & 0 & 0
\end{array}\right]\left[(2 \lambda+1)\left(2 \lambda^{\prime}+1\right)\right]^{1 / 2} \\
& \times\left[\left(2 K_{j}+1\right)\left(2 t^{\prime}+1\right)(2 t+1)(2 j+1)\left(2 j^{\prime}+1\right)\right]^{1 / 2} \\
& \times\left\{\begin{array}{ccc}
J_{1} & K_{1} & J_{1} \\
t^{\prime} & K_{q} & t \\
J^{\prime} & K & J
\end{array}\right\}\left\{\begin{array}{ccc}
J_{2} & K_{2} & J_{2} \\
j^{\prime} & K_{j} & j \\
J^{\prime} & K & J
\end{array}\right\}\left\{\begin{array}{ccc}
\lambda^{\prime} & K_{\lambda} \lambda \\
s & K_{s} & s \\
j^{\prime} & K_{j} & j
\end{array}\right\} .
\end{aligned}
$$

In (11), the operator is defined as

$$
Q_{m_{t}}^{(t)}=\mathrm{i}^{t} j_{t}(q r) C_{m_{t}}^{(t)}(\hat{r})
$$

where $C_{m_{t}}^{(t)}(\hat{r})$ is the operator of spherical function [11], and the constant is $C=1 / \pi$. Here the $1 / 2$ coming from averaging over the spin projections of projectile electron is also taken into account.

When the fast scattered electron is not detected, the integration of (10) over the angles of this electron can be performed. It can be changed by the integration over the momentum transferred to an atom. Then

$$
\begin{aligned}
& \frac{\mathrm{d}^{2} \sigma\left(\alpha_{1} J_{1} M_{1} \mathbf{q} \rightarrow \alpha_{2} J_{2} M_{2} \mathbf{p} m\right)}{\mathrm{d} \Omega \mathrm{d} \varepsilon} \\
& =\int \mathrm{d} \Omega_{q} \frac{\left.\mathrm{d}^{3} \sigma\left(\alpha_{1} J_{1} M_{1} \mathbf{q} \rightarrow \alpha_{2} J_{2} M_{2} \mathbf{p} m\right)\right)}{\mathrm{d} \Omega \mathrm{d} \Omega_{q} \mathrm{~d} \varepsilon}=\frac{2 \pi k C}{k_{1}^{2}} \\
& \times \sum_{\substack{K_{1}, K_{2}, K_{q}, K_{\lambda}, K_{s}, K_{j}, K}} B^{\text {B.ion. }}\left(K_{1}, K_{2}, K_{q}, K_{\lambda}, K_{s}, K_{j}, K\right) \\
& \times \sum_{\substack{N_{1}, N_{2}, N_{q}, N_{\lambda}, N_{s}, N_{j}, N}} 4 \pi Y_{K_{\lambda} N_{\lambda}}(\hat{p}) Y_{K_{q} N_{q}}^{*}(\hat{q}) \\
& \times\left[\begin{array}{lll}
K_{1} & K_{q} & K \\
N_{1} & N_{q} & N
\end{array}\right]\left[\begin{array}{lll}
K_{\lambda} & K_{s} & K_{j} \\
N_{\lambda} & N_{s} & N_{j}
\end{array}\right]\left[\begin{array}{lll}
K_{2} & K_{j} & K \\
N_{2} & N_{j} & N
\end{array}\right] \\
& \times T_{N_{1}}^{* K_{1}}\left(\hat{J}_{1}\right) T_{N_{2}}^{K_{2}}\left(\hat{J}_{2}\right) T_{N_{s}}^{K_{s}}(\hat{s}),
\end{aligned}
$$

where

$$
\begin{aligned}
& B^{\text {B.ion. }}\left(K_{1}, K_{2}, K_{q}, K_{\lambda}, K_{s}, K_{j}, K\right) \\
& =\int_{q_{\min }}^{q_{\max }} \frac{\mathrm{d} q}{q^{3}} \mathcal{B}^{\text {B.ion. }}\left(K_{1}, K_{2}, K_{q}, K_{\lambda}, K_{s}, K_{j}, K\right), \\
& q_{\max }=\left(2 \varepsilon_{1}\right)^{1 / 2}+\left[2\left(\varepsilon_{1}-I_{p}-\varepsilon\right)\right]^{1 / 2}
\end{aligned}
$$




$$
q_{\min }=\left(2 \varepsilon_{1}\right)^{1 / 2}-\left[2\left(\varepsilon_{1}-I_{p}-\varepsilon\right)\right]^{1 / 2} .
$$

The total ionization cross-section can be obtained by integration of (13) over the angles of the emitted electron and its energies from 0 up to $\varepsilon_{1}-I_{p}$, where $I_{p}$ is the ionization potential of an atom.

The expression (14) represents the general case of the cross-section describing the ionization of polarized atoms by non-polarized electrons and enabling one to obtain information on the angular distributions, angular correlations, and spin polarization of the emitted electron in plane wave Born approximation. It can be used for the derivation of some special expressions applicable for the specific experimental conditions.

\section{Special cases}

\subsection{Total cross-section for the ionization of non-polarized atoms}

To obtain the total cross-section for the ionization of non-polarized atoms by fast non-polarized electrons, one needs to do the integration over the angles of the slow emitted electron and the summation over the magnetic components of all angular momenta. The following summation and integration formulas [4] make it easy:

$$
\sum_{M} T_{N}^{K}(J, J, M \mid \hat{J})=\delta(K, 0) \delta(N, 0),
$$

$$
\int_{0}^{\pi} \int_{0}^{2 \pi} \sin \theta \mathrm{d} \theta \mathrm{d} \phi Y_{K N}(\theta, \phi)=\sqrt{4 \pi} \delta(K, 0) \delta(N, 0) .
$$

Then the expression for the total cross-section is as follows:

$$
\begin{aligned}
& \frac{\mathrm{d} \sigma\left(\alpha_{1} J_{1} \rightarrow \alpha_{2} J_{2} \varepsilon\right)}{\mathrm{d} \varepsilon}=\frac{1}{2 J_{1}+1} \\
& \quad \times \int \mathrm{d} \Omega \sum_{M_{1}, M_{2}, m} \frac{\mathrm{d}^{2} \sigma\left(\alpha_{1} J_{1} M_{1} \rightarrow \alpha_{2} J_{2} M_{2} \mathbf{p} m\right)}{\mathrm{d} \Omega \mathrm{d} \varepsilon} \\
& =\frac{4 \pi^{2} k C}{\varepsilon_{1}\left(2 J_{1}+1\right)} B^{\text {B.ion. }}(0,0,0,0,0,0,0)
\end{aligned}
$$

where $C=1 / \pi$ and

$$
\begin{aligned}
& B^{\text {B.ion. }}(0,0,0,0,0,0,0)=\int_{q_{\min }}^{q_{\max }} \frac{\mathrm{d} q}{q^{3}} \sum_{\lambda, j, J, t}(2 J+1) \\
& \times\left|\left\langle\alpha_{2} J_{2} \varepsilon \lambda(j) J\left\|Q^{(t)}\right\| \alpha_{1} J_{1}\right\rangle\right|^{2} .
\end{aligned}
$$

Often the total cross-section integrated over all the energies of emitted electron is used. Its expression obtained from (13) is

$$
\begin{aligned}
& \sigma\left(\alpha_{1} J_{1} \rightarrow \alpha_{2} J_{2}\right)=\int_{0}^{\varepsilon_{1}-I_{p}} \mathrm{~d} \varepsilon \frac{\mathrm{d} \sigma\left(\alpha_{1} J_{1} \rightarrow \alpha_{2} J_{2} \varepsilon\right)}{\mathrm{d} \varepsilon} \\
& =\frac{4 \pi}{\varepsilon_{1}\left(2 J_{1}+1\right)} \int_{0}^{\varepsilon_{1}-I_{p}} \mathrm{~d} \varepsilon B^{\text {B.ion. }}(0,0,0,0,0,0,0) \\
& =\frac{4 \pi}{\varepsilon_{1}\left(2 J_{1}+1\right)} \int_{0}^{\varepsilon_{1}-I_{p}} \mathrm{~d} \varepsilon \int_{q_{\min }}^{q_{\max }} \frac{\mathrm{d} q}{q^{3}} \sum_{\lambda, j, J, t}(2 J+1) \\
& \quad \times\left|\left\langle\alpha_{2} J_{2} \varepsilon \lambda(j) J\left\|Q^{(t)}\right\| \alpha_{1} J_{1}\right\rangle\right|^{2} .
\end{aligned}
$$

Here $I_{p}$ is the ionization energy, and the integration over $k$ present in (19) is performed after replacement $\mathrm{d} \varepsilon=k \mathrm{~d} k$.

\subsection{Angular distribution of the emitted electrons from non-polarized atoms}

The expression for the differential cross-section describing the angular distribution of emitted electron from non-polarized atoms, when the polarization of electron and ion is not registered, can be obtained by performing the summation of Eq. (13) over the magnetic components $M_{2}$ and $m$ and averaging over the states of an atom. It acquires the form

$$
\begin{aligned}
& \frac{\mathrm{d}^{2} \sigma\left(\alpha_{1} J_{1} \mathbf{q} \rightarrow \alpha_{2} J_{2} \mathbf{p}\right)}{\mathrm{d} \varepsilon \mathrm{d} \Omega}=\frac{1}{2 J_{1}+1} \\
& \times \sum_{M_{1}, M_{2}, m} \frac{\mathrm{d}^{2} \sigma\left(\alpha_{1} J_{1} M_{1} \mathbf{q} \rightarrow \alpha_{2} J_{2} M_{2} \mathbf{p} m\right)}{\mathrm{d} \Omega \mathrm{d} \varepsilon} \\
& =\frac{\pi k C}{\varepsilon_{1}\left(2 J_{1}+1\right)} \sum_{K_{\lambda}} B^{\text {B.ion. }}\left(0,0, K_{\lambda}, K_{\lambda}, 0, K_{\lambda}, K_{\lambda}\right) \\
& \quad \times \sum_{N_{\lambda}} 4 \pi Y_{K_{\lambda} N_{\lambda}}(\hat{p}) Y_{K_{\lambda} N_{\lambda}}^{*}(\hat{q}) .
\end{aligned}
$$

Taking into account the axial symmetry of the ionization process and by choosing the laboratory $z$ axis along the direction of transferred momentum $\mathbf{q}$, the expression (22) becomes simpler:

$$
\begin{aligned}
& \frac{\mathrm{d}^{2} \sigma\left(\alpha_{1} J_{1} \rightarrow \alpha_{2} J_{2} \mathbf{p}\right)}{\mathrm{d} \varepsilon \mathrm{d} \Omega}=\frac{1}{4 \pi} \frac{\mathrm{d} \sigma\left(\alpha_{1} J_{1} \rightarrow \alpha_{2} J_{2} \varepsilon\right)}{\mathrm{d} \varepsilon} \\
& \times\left[1+\sum_{K_{\lambda}>0} \beta_{K_{\lambda}} P_{K_{\lambda}}(\cos \theta)\right]
\end{aligned}
$$


where

$$
\beta_{K_{\lambda}}=\frac{\left(2 K_{\lambda}+1\right) B^{\text {B.ion. }}\left(0,0, K_{\lambda}, K_{\lambda}, 0, K_{\lambda}, K_{\lambda}\right)}{B^{\text {B.ion. }}(0,0,0,0,0,0,0)}
$$

is the asymmetry parameter of the angular distribution of emitted electron. The angle $\theta$ is measured from the direction of the transferred momentum q. For a given initial energy $\varepsilon_{1}$ and an energy loss $\Delta E$ of the initial electron, its scattering angle $\theta$ is related to the momentum transfer $q$ by the relation

$$
\cos \theta=\frac{2 \varepsilon_{1}-\Delta E-q^{2}}{2\left[\varepsilon_{1}\left(\varepsilon_{1}-\Delta E\right)\right]^{1 / 2}} .
$$

\subsection{Total cross-section for the polarized atoms}

The expression for the total ionization cross-section from polarized atoms is as follows:

$$
\begin{aligned}
& \frac{\mathrm{d} \sigma\left(\alpha_{1} J_{1} M_{1} \rightarrow \alpha_{2} J_{2} \varepsilon\right)}{\mathrm{d} \varepsilon} \\
& =\int \mathrm{d} \Omega \sum_{M_{2}, m} \frac{\mathrm{d}^{2} \sigma\left(\alpha_{1} J_{1} M_{1} \rightarrow \alpha_{2} J_{2} M_{2} \mathbf{p} m\right)}{\mathrm{d} \Omega \mathrm{d} \varepsilon} \\
& =\frac{4 \pi^{2} k C}{\varepsilon_{1}} \sum_{K, N} B^{\text {B.ion. }}(K, 0, K, 0,0,0,0)(-1)^{K-N} \\
& \quad \times\left[\frac{4 \pi}{2 K+1}\right]^{1 / 2} Y_{K N}^{*}\left(\theta_{q}, \phi_{q}\right) T_{N}^{* K}\left(\hat{J}_{1}\right) .
\end{aligned}
$$

By choosing the $z$ axis along the direction of the transferred momentum $\mathbf{q}$ the expression (26) becomes simpler:

$$
\begin{aligned}
& \frac{\mathrm{d} \sigma\left(\alpha_{1} J_{1} M_{1} \rightarrow \alpha_{2} J_{2} \varepsilon\right)}{\mathrm{d} \varepsilon} \\
= & \frac{4 \pi^{2} k C}{\varepsilon_{1}} \sum_{K} B^{\text {B.ion. }}(K, 0, K, 0,0,0,0)(-1)^{J_{1}-M_{1}+K} \\
& \times\left[\frac{4 \pi}{2 J_{1}+1}\right]^{1 / 2}\left[\begin{array}{ccc}
J_{1} & J_{1} & K \\
M_{1}-M_{1} & 0
\end{array}\right] Y_{K 0}^{*}(\theta, \phi) \\
= & \frac{4 \pi^{2} k C}{\varepsilon_{1}} \sum_{K} B^{\text {B.ion. }}(K, 0, K, 0,0,0,0)(-1)^{J_{1}-M_{1}+K} \\
& \times\left[\frac{2 K+1}{2 J_{1}+1}\right]^{1 / 2}\left[\begin{array}{ccc}
J_{1} & J_{1} & K \\
M_{1}-M_{1} & 0
\end{array}\right] P_{K}(\cos \theta) .
\end{aligned}
$$

The magnetic dichroism is defined by the following formula:

$$
\Delta=\frac{\sigma(J M)-\sigma(J-M)}{\sigma(J M)+\sigma(J-M)},
$$

where $\sigma(J M)=\mathrm{d} \sigma\left(\alpha_{1} J_{1} M_{1} \rightarrow \alpha_{2} J_{2} \varepsilon\right) / \mathrm{d} \varepsilon$. The magnetic dichroism in the ionization of polarized atoms by non-polarized electrons acquires the following expression:

$$
\begin{gathered}
\Delta=\frac{-\sum_{K_{\text {odd }}} B^{\text {B.ion. }}\left(K_{\text {odd }}, 0, K_{\text {odd }}, 0,0,0,0\right)}{\sum_{K_{\text {even }}} B^{\text {B.ion. }}\left(K_{\text {even }}, 0, K_{\text {even }}, 0,0,0,0\right)} \\
\times \frac{\sqrt{2 K_{\text {odd }}+1}\left[\begin{array}{ccc}
J_{1} & J_{1} & K_{\text {odd }} \\
M_{1}-M_{1} & 0
\end{array}\right] P_{K_{\text {odd }}}(\cos \theta)}{\sqrt{2 K_{\text {even }}+1}\left[\begin{array}{ccc}
J_{1} & J_{1} & K_{\text {even }} \\
M_{1}-M_{1} & 0
\end{array}\right] P_{K_{\text {even }}}(\cos \theta)}
\end{gathered}
$$

It simplifies in the case of the atoms polarized along the direction of transferred momentum $\mathbf{q}$

$$
\begin{aligned}
\Delta & =\frac{-\sum_{K_{\text {odd }}} B^{\text {B.ion. }}\left(K_{\text {odd }}, 0, K_{\text {odd }}, 0,0,0,0\right)}{\sum_{K_{\text {even }}} B^{\text {B.ion. }}\left(K_{\text {even }}, 0, K_{\text {even }}, 0,0,0,0\right)} \\
& \times \frac{\sqrt{2 K_{\text {odd }}+1}\left[\begin{array}{lll}
J_{1} & J_{1} & K_{\text {odd }} \\
J_{1}-J_{1} & 0
\end{array}\right]}{\sqrt{2 K_{\text {even }}+1}\left[\begin{array}{lll}
J_{1} & J_{1} & K_{\text {even }} \\
J_{1}-J_{1} & 0
\end{array}\right]} .
\end{aligned}
$$

The parameter (30) of the magnetic dichroism in the case of $J_{1}=1 / 2$ is

$$
\begin{aligned}
& \Delta=\frac{-B^{\text {B.ion. }}(1,0,1,0,0,0,0) \sqrt{3}\left[\begin{array}{lll}
1 / 2 & 1 / 2 & 1 \\
1 / 2-1 / 2 & 0
\end{array}\right]}{B^{\text {B.ion. }}(0,0,0,0,0,0,0) 2^{-1 / 2}} \\
& =\frac{-\sqrt{3} B^{\text {B.ion. }}(1,0,1,0,0,0,0)}{B^{\text {B.ion. }}(0,0,0,0,0,0,0)} .
\end{aligned}
$$

For $J_{1}=1$, it is

$$
\begin{aligned}
\Delta= & \frac{-3 B^{\text {B.ion. }}(1,0,1,0,0,0,0)}{\left[\sqrt{2} B^{\text {B.ion. }}(0,0,0,0,0,0,0)\right.} . \\
& \left.+\sqrt{5} B^{\text {B.ion. }}(2,0,2,0,0,0,0)\right]
\end{aligned}
$$




\subsection{Angular distribution of the emitted electrons from polarized atoms}

In the case of the ionization of polarized atoms, the differential cross-section depending on the angular distribution of emitted electrons can be written as follows:

$$
\begin{aligned}
& \frac{\mathrm{d}^{2} \sigma\left(\alpha_{1} J_{1} M_{1} \mathbf{q} \rightarrow \alpha_{2} J_{2} \mathbf{p}\right)}{\mathrm{d} \varepsilon \mathrm{d} \Omega} \\
= & \sum_{M_{2}, m} \frac{\mathrm{d}^{2} \sigma\left(\alpha_{1} J_{1} M_{1} \rightarrow \alpha_{2} J_{2} M_{2} \mathbf{p} m\right)}{\mathrm{d} \Omega \mathrm{d} \varepsilon} \\
= & \frac{\pi k C}{\varepsilon_{1}} \sum_{K_{1}, K_{q}, K_{\lambda}} B^{\text {B.ion. }}\left(K_{1}, 0, K_{q}, K_{\lambda}, 0, K_{\lambda}, K_{\lambda}\right) \\
& \times \sum_{N_{1}, N_{\lambda}, N_{q}} 4 \pi\left[\begin{array}{c}
K_{1} K_{q} K_{\lambda} \\
N_{1} N_{q} N_{\lambda}
\end{array}\right] Y_{K_{\lambda} N_{\lambda}}(\hat{p}) Y_{K_{q} N_{q}}(\hat{q}) \\
& \times T^{* K_{1}}\left(\hat{J}_{1}\right) .
\end{aligned}
$$

In the case of the $z$ axis coinciding with the direction of transferred momentum $\mathbf{q}$, it reduces to

$$
\begin{aligned}
& \frac{\mathrm{d}^{2} \sigma\left(\alpha_{1} J_{1} M_{1} \rightarrow \alpha_{2} J_{2} \mathbf{p}\right)}{\mathrm{d} \varepsilon \mathrm{d} \Omega}=\frac{4 \pi^{2} k C}{\varepsilon_{1}} \\
& \times \sum_{K_{1}, K_{q}, K_{\lambda}, N}\left[\begin{array}{ccc}
K_{1} & K_{q} & K_{\lambda} \\
N & 0 & N
\end{array}\right] Y_{K_{\lambda} N}(\hat{p}) Y_{K_{1} N}^{*}\left(\hat{J}_{1}\right) \\
& \quad \times\left[\frac{2 K_{q}+1}{2 J_{1}+1}\right]^{1 / 2}(-1)^{J_{1}-M_{1}}\left[\begin{array}{ccc}
J_{1} & J_{1} & K_{1} \\
M_{1}-M_{1} & 0
\end{array}\right] \\
& \quad \times B^{\text {B.ion. }}\left(K_{1}, 0, K_{q}, K_{\lambda}, 0, K_{\lambda}, K_{\lambda}\right) \\
& =\frac{4 \pi^{2} k C}{\varepsilon_{1}} \sum_{K_{\lambda}, N} \beta_{K_{\lambda} N}\left(\hat{J}_{1}\right)\left[\frac{4 \pi}{2 K_{\lambda}+1}\right]^{1 / 2} Y_{K_{\lambda} N}(\hat{p}),
\end{aligned}
$$

where

$$
\begin{gathered}
\beta_{K_{\lambda} N}\left(\hat{J}_{1}\right)=\sum_{K_{1}, K_{q}} B^{\text {B.ion. }}\left(K_{1}, 0, K_{q}, K_{\lambda}, 0, K_{\lambda}, K_{\lambda}\right) \\
\times\left[\frac{\left(2 K_{\lambda}+1\right)\left(2 K_{q}+1\right)}{4 \pi\left(2 J_{1}+1\right)}\right]^{1 / 2}\left[\begin{array}{ccc}
K_{1} K_{q} K_{\lambda} \\
N & 0 & N
\end{array}\right] \\
\times(-1)^{J_{1}-M_{1}}\left[\begin{array}{ccc}
J_{1} & J_{1} & K_{1} \\
M_{1}-M_{1} & 0
\end{array}\right] Y_{K_{1} N}^{*}\left(\hat{J}_{1}\right)
\end{gathered}
$$

are the parameters of the asymmetry of the angular distribution of emitted electrons containing the information about the polarization state of atoms.

\subsection{The alignment of ionized atoms}

The alignment of ionized atoms describes their state of polarization and can be measured in the second step processes (those follow the first step of ionization). As a rule, the alignment of the ionized atom strongly affects the parameters of the angular distribution and polarization of Auger electrons and fluorescence radiation. The expression (13) written in the form of the multipole expansion of the final state of an ion is more suitable for the investigation of alignment in the second step processes. Applying the procedure described in Ref. [2] it can be written in the following form:

$$
\begin{aligned}
& \frac{\mathrm{d}^{2} \sigma_{K_{2} N_{2}}\left(\alpha_{1} J_{1} M_{1} \mathbf{q} \rightarrow \alpha_{2} J_{2} \mathbf{p} m\right)}{\mathrm{d} \Omega \mathrm{d} \varepsilon}=\frac{\pi k C}{\varepsilon_{1}} \\
& \times \sum_{\substack{K_{1}, K_{q}, K_{\lambda}, K_{s}, K_{j}, K}} B^{\text {B.ion. }}\left(K_{1}, K_{2}, K_{q}, K_{\lambda}, K_{s}, K_{j}, K\right) \\
& \times \sqrt{2 K_{2}+1} \sum_{\substack{N_{1}, N_{q}, N_{\lambda}, N_{s}, N_{j}, N}}\left[\begin{array}{l}
K_{1} K_{q} K \\
N_{1} N_{q} N
\end{array}\right]\left[\begin{array}{l}
K_{\lambda} K_{s} K_{j} \\
N_{\lambda} N_{s} N_{j}
\end{array}\right] \\
& \times\left[\begin{array}{c}
K_{2} K_{j} K \\
N_{2} N_{j} N
\end{array}\right] \sqrt{4 \pi} Y_{K_{\lambda} N_{\lambda}}(\hat{p}) \sqrt{4 \pi} Y_{K_{q} N_{q}}^{*}(\hat{q}) \\
& \times T^{* K_{1}}\left(\hat{J}_{1}\right) T_{N_{s}}^{K_{s}}(\hat{s}) .
\end{aligned}
$$

By using (36) the expression for the cross-section describing the alignment of ionized atoms in the case of the ionization of non-polarized atoms by non-polarized fast electrons can be written as follows:

$$
\begin{aligned}
& \frac{\mathrm{d} \sigma\left(\alpha_{1} J_{1} \mathbf{q} \rightarrow \alpha_{1} J_{2} \varepsilon\right)}{\mathrm{d} \varepsilon}=\sum_{K_{2}, N_{2}} \frac{1}{2 J_{1}+1} \\
& \quad \times \int \mathrm{d} \Omega \sum_{M_{1}, m} \frac{\mathrm{d}^{2} \sigma_{K_{2} N_{2}}\left(\alpha_{1} J_{1} M_{1} \mathbf{q} \rightarrow \alpha_{2} J_{2} \mathbf{p} m\right)}{\mathrm{d} \Omega \mathrm{d} \varepsilon} \\
& =\frac{4 \pi^{2} k C}{\varepsilon_{1}\left(2 J_{1}+1\right)} \sum_{K_{2}, N_{2}} \sqrt{2 K_{2}+1} \sqrt{4 \pi} Y_{K_{2} N_{2}}^{*}(\hat{q}) \\
& \quad \times B^{\text {B.ion. }}\left(0, K_{2}, K_{2}, 0,0,0, K_{2}\right) .
\end{aligned}
$$

In the case of $z$ axis chosen along $\mathbf{q}$, Eq. (36) becomes very simple:

$$
\frac{\mathrm{d} \sigma\left(\alpha_{1} J_{1} \rightarrow \alpha_{1} J_{2} \varepsilon\right)}{\mathrm{d} \varepsilon}=\frac{4 \pi^{2} k C}{\varepsilon_{1}\left(2 J_{1}+1\right)}
$$


$\times \sum_{K_{2}}\left(2 K_{2}+1\right) B^{\text {B.ion. }}\left(0, K_{2}, K_{2}, 0,0,0, K_{2}\right)$.

The inspection of the expression for $B^{\text {B.ion. }}$ (14) allows us to conclude that $K_{2}$ must be even. The alignment describes $K_{2}=2$ and higher terms. Therefore, the expression (38) can be rewritten in the form

$$
\begin{aligned}
& \frac{\mathrm{d} \sigma\left(\alpha_{1} J_{1} \rightarrow \alpha_{1} J_{2} \varepsilon\right)}{\mathrm{d} \varepsilon}=\sigma_{0}\left(\alpha_{1} J_{1} \rightarrow \alpha_{1} J_{2} \varepsilon\right) \\
& \times\left[1+\sum_{K_{2}>0, \text { even }} A_{K_{2}}\right],
\end{aligned}
$$

where

$$
\begin{gathered}
A_{K_{2}}=\frac{\left(2 K_{2}+1\right) B^{\text {B.ion. }}\left(0, K_{2}, K_{2}, 0,0,0, K_{2}\right)}{B^{\text {B.ion. }}(0,0,0,0,0,0,0)} \\
\sigma_{0}\left(\alpha_{1} J_{1} \rightarrow \alpha_{1} J_{2} \varepsilon\right)=\frac{4 \pi^{2} k C}{\varepsilon_{1}\left(2 J_{1}+1\right)} \\
\quad \times B^{\text {B.ion. }}(0,0,0,0,0,0,0) .
\end{gathered}
$$

Similar procedures are applied for the derivation of the cross-section describing the alignment of ions created in the ionization of polarized atoms:

$$
\begin{aligned}
& \frac{\mathrm{d} \sigma\left(\alpha_{1} J_{1} M_{1} \mathbf{q} \rightarrow \alpha_{1} J_{2} \varepsilon\right)}{\mathrm{d} \varepsilon} \\
& =\sum_{K_{2}, N_{2}} \int \mathrm{d} \Omega \sum_{m} \frac{\mathrm{d}^{2} \sigma_{K_{2} N_{2}}\left(\alpha_{1} J_{1} M_{1} \mathbf{q} \rightarrow \alpha_{2} J_{2} \mathbf{p} m\right)}{\mathrm{d} \Omega \mathrm{d} \varepsilon} \\
& =\frac{4 \pi^{2} k C}{\varepsilon_{1}} \sum_{\substack{K_{2}, N_{2}, K_{1} \\
N_{1}, K_{q}, N_{q}}} \sqrt{2 K_{2}+1}\left[\begin{array}{c}
K_{1} K_{q} K_{2} \\
N_{1} N_{q} N_{2}
\end{array}\right] \\
& \quad \times \sqrt{4 \pi} B^{\text {B.ion. }\left(K_{1}, K_{2}, K_{q}, 0,0,0, K_{2}\right)} \\
& \quad \times Y_{K_{q} N_{q}}(\hat{q}) T^{* K_{1}}\left(\hat{J}_{1}\right) .
\end{aligned}
$$

It also simplifies for the case of the $z$ axis chosen along $\mathbf{q}$ and is equal to

$$
\begin{aligned}
& \frac{\mathrm{d} \sigma\left(\alpha_{1} J_{1} M_{1} \rightarrow \alpha_{1} J_{2} \varepsilon\right)}{\mathrm{d} \varepsilon} \\
& =\frac{4 \pi^{2} k C}{\varepsilon_{1}} \sum_{K_{2}, K_{1}, K_{q}, N} B^{\text {B.ion. }}\left(K_{1}, K_{2}, K_{q}, 0,0,0, K_{2}\right) \\
& \quad \times \sqrt{2 K_{2}+1}\left[\begin{array}{crr}
K_{1} & K_{q} & K_{2} \\
N & 0 & N
\end{array}\right]\left[\frac{\left(2 K_{q}+1\right) 4 \pi}{2 J_{1}+1}\right]^{1 / 2}
\end{aligned}
$$

$$
\begin{aligned}
& \times(-1)^{J_{1}-M_{1}}\left[\begin{array}{ccc}
J_{1} & J_{1} & K_{1} \\
M_{1}-M_{1} & 0
\end{array}\right] Y_{K_{1} N}^{*}\left(\hat{J}_{1}\right) \\
= & \frac{4 \pi^{2} k C}{\varepsilon_{1}} \sum_{K_{2}} A_{K_{2}}\left(\hat{J}_{1}\right),
\end{aligned}
$$

where

$$
\begin{aligned}
& A_{K_{2}}\left(\hat{J}_{1}\right)=\sum_{K_{1}, K_{q}, N} B^{\text {B.ion. }}\left(K_{1}, K_{2}, K_{q}, 0,0,0, K_{2}\right) \\
& \quad \times(-1)^{J_{1}-M_{1}} Y_{K_{1} N}^{*}\left(\hat{J}_{1}\right)\left[\begin{array}{ccc}
J_{1} & J_{1} & K_{1} \\
M_{1}-M_{1} & 0
\end{array}\right]
\end{aligned}
$$$$
\times\left[\begin{array}{ccc}
K_{1} & K_{q} & K_{2} \\
N & 0 & N
\end{array}\right]\left[\frac{\left(2 K_{2}+1\right)\left(2 K_{q}+1\right) 4 \pi}{2 J_{1}+1}\right]^{1 / 2}
$$

is the parameter of the alignment depending on the polarization state of an atom.

\section{References}

[1] S.A. Kazantsev and J.-C. Henoux, Polarization Spectroscopy of Ionized Atoms (Kluwer, Dordrecht, Boston, London, 1995).

[2] A. Kupliauskiene, Atomic theory methods for the polarization of photon and electron interactions with atoms, Lithuanian J. Phys. 44, 199-218 (2004).

[3] A. Kupliauskienė, N. Rakštikas, and V. Tutlys, General expression of the photoionization cross section of an atom in polarized $L S$ state, Lithuanian J. Phys. 40, 311-320 (2000).

[4] A. Kupliauskienè, N. Rakštikas, and V. Tutlys, Polarization studies in the photoionization of atoms using a graphical technique, J. Phys. B 34, 1783-1803 (2001).

[5] A. Kupliauskienè and K. Glemža, General expression for ionization cross-section of polarized atoms by polarized electrons, Lithuanian J. Phys. 43, 89-97 (2003).

[6] A. Kupliauskienè, Photoexcitation of polarized atoms by polarized radiation, Lithuanian J. Phys. 44, 17-26 (2004).

[7] V.V. Balashov, A.N. Grum-Grzhimailo, and N.M. Kabachnik, Polarization and Correlation Phenomena in Atomic Collisions. A Practical Theory Course (Kluwer, New York, 2000).

[8] S.C. McFarlane, The polariztion of characteristic $x$ radiation excited by electron impact, J. Phys. B 5, 19061915 (1972).

[9] E.G. Berezhko, N.M. Kabachnik, and V.V. Sizov, The theory of coincidence experiments on electron impact ionization of inner atomic shells, J. Phys. B 11, 18191832 (1978).

[10] A. Dorn, A. Elliott, J. Lower, E. Weigold, and J. Berakdar, Orientational dichroism in the electron-impact 
ionization of laser-oriented atomic sodium, Phys. Rev. Lett. 80, 257-260 (1998).

[11] A.P. Jucys and A.A. Bandzaitis, Theory of Angular
Momentum in Quantum Mechanics (Mintis, Vilnius, 1965) [in Russian].

\title{
POLIARIZUOTU ATOMU JONIZACIJOS GREITAISIAIS ELEKTRONAIS TEORINIS TYRIMAS
}

\author{
K. Glemža ${ }^{a}$ and A. Kupliauskiené ${ }^{b}$ \\ ${ }^{a}$ Vilniaus universitetas, Vilnius, Lietuva \\ ${ }^{\mathrm{b}}$ VU Teorines fizikos ir astronomijos institutas, Vilnius, Lietuva
}

\section{Santrauka}

Atomu ir jonu jonizacija - vienas svarbiausiu procesu plazmoje. Esant kryptingam krūvininku judèjimui, pvz. tokamako plazmoje, galima jonų būsenų savaiminè poliarizacija. Dažniausiai atsiranda savaiminis rikiavimas, kai būsenos, aprašomos vienodo didumo bet priešingu ženklų pilnutinio judèjimo kiekio momento $J$ projekcijomis $M$, užpildomos vienodai. Tokiu jonų elektromagnetinè spinduliuotè būna poliarizuota. Iš spinduliuotès poliarizacijos galima nustatyti elektronu pasiskirstymo pagal greičius funkcijos nukrypimo nuo Maksvelo funkcijos mastą.
Naudojant atomo teorijos metodus, pritaikytus poliarizacijos reiškiniams tirti, ir Borno artinio operatoriaus panašumą i fotojonizacijos operatoriu, surasta bendroji poliarizuoto atomo jonizacijos greitaisiais nepoliarizuotais elektronais diferencialinio skerspjūvio išraiška. Ji panaudota lètesniojo elektrono kampinio pasiskirstymo ir jonizuoto atomo rikiavimo parametrams surasti, kai jonizuojami poliarizuoti ir nepoliarizuoti atomai, bei poliarizuoto atomo jonizacijos pilnutinio skerspjūvio magnetiniam dichroizmui aprašyti. 